\title{
Impedance Eduction for Multisegment Liners
}

\author{
M. G. Jones; $\quad$ W. R. Watson† $\quad$ D. M. Nark $\ddagger$ and B. M. Howerton ${ }^{\S}$ \\ NASA Langley Research Center, Hampton, VA 23681
}

\begin{abstract}
This paper explores the validity of an indirect method for impedance eduction of multisegment liners. This is accomplished via results obtained with two uniform liners and one two-segment liner, where each segment is constructed to match the geometry of one of the uniform liners. Each uniform liner is evaluated using direct and indirect impedance eduction methods. An indirect impedance eduction method is used to educe the impedance for each segment of the two-segment liner, and the results are compared with those educed for the uniform liners. These impedance spectra are shown to compare favorably for the majority of test conditions. Poorer comparisons are achieved for those test conditions where one segment of the two-segment liner provides little attenuation. Poor attenuation is a wellknown cause for impedance eduction difficulties. Overall, this multisegment impedance eduction method offers the potential to study complicated liners in a more efficient manner (i.e., without the requirement to build and test separate liners to duplicate each unique segment of the multisegment liner). More detailed studies are required to further validate this tool, and are intended to be the focus of future research.
\end{abstract}

\section{Introduction}

One of the dominant contributors to aircraft noise is fan noise that emanates from both the inlet and the aftfan duct of engine nacelles. Conventional perforate-over-honeycomb liners are typically installed in the inner walls of engine nacelles to absorb this objectionable noise before it emanates to communities surrounding airports. These liners have enabled commercial aircraft to meet increasingly stringent noise constraints imposed by the Federal Aviation Administration and International Civil Aviation Organization. However, improved absorption is needed as these constraints are continually tightened.

To achieve improved sound absorption, a number of novel concepts are being considered that are enabled by additive manufacturing (3D printing). One option that has been the focus of study at NASA Langley is the multisegment (multizone) liner. For fabrication convenience, these are often variable-depth liners, ${ }^{1,2}$ where each axial segment is comprised of chambers with different depths. Whereas these multisegment liners provide the capability of increased broadband sound absorption, they also present unique challenges. One such challenge is the need for an approach to simultaneously educe the impedance of each axial segment.

A number of impedance eduction approaches have been considered, including in situ, ${ }^{3}$ direct, ${ }^{4}$ and indirect ${ }^{5}$ methods. The in situ method usually employs two microphones, one at the back plate and the other at the facesheet, to educe the impedance of the liner in the immediate vicinity of the microphones (local impedance). As such, this method is a viable candidate for determining the impedance of each liner segment.

The direct methods in use at NASA Langley are based on acoustic pressure measurements with microphones along the length of the liner on the wall opposite the liner. These acoustic pressure measurements are used to obtain axial propagation constants that are used in the impedance boundary condition to directly determine the liner impedance. These direct methods assume the liner to be uniform with a constant impedance. Thus, if an individual segment of the multisegment liner is sufficiently long that a suitable number of microphones can be mounted on the wall directly opposite the liner, these methods should be suitable

\footnotetext{
* Senior Research Scientist, Research Directorate, Structural Acoustics Branch, AIAA Associate Fellow.

†Senior Research Scientist, Research Directorate, Computational AeroSciences Branch, AIAA Associate Fellow.

¥Senior Research Scientist, Research Directorate, Structural Acoustics Branch, AIAA Associate Fellow.

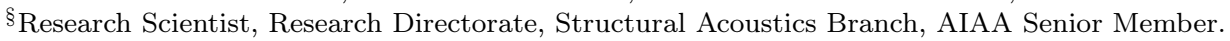


for eduction of the impedance for this segment. If these conditions cannot be achieved, these methods are not appropriate for multisegment liners.

The indirect impedance eduction method, as utilized at NASA, is ideally suited for the multisegment liner application. This method is based on acoustic pressure measurements over the length of duct that includes the liner and hard wall sections upstream and downstream of the liner. These acoustic pressures are used to construct an objective function whose minimum occurs at the impedance of the liner. An optimization method is typically used to obtain the impedance that minimizes this objective function. Although this method has traditionally been used for uniform, constant-impedance liners, it is easily adapted to account for multisegment liners.

The current paper seeks to validate this indirect method for impedance eduction with a two-segment liner. This is achieved with the use of two uniform liners and a two-segment liner, where the two segments are designed to be identical in construction to each of the two uniform liners. Two impedance eduction methods, one direct and one indirect, are briefly described in Section II. Section III provides a description of the test liners and the test rig used for the validation process. Key results are discussed in Section IV, and some concluding remarks are provided in Section V.

\section{Analysis}

Two methods are employed for impedance eduction, one indirect and one direct. The primary purpose of this study is to validate an indirect method to simultaneously educe the impedances for each segment of a two-segment liner. This is achieved via comparison of impedance spectra educed using the direct method for two uniform-impedance liners with those educed for the two-segment liner using the indirect method.

\section{A. Convected Helmholtz Equation (CHE) Method}

A description of the indirect impedance eduction method (referred to as the CHE method) has been provided by Watson, ${ }^{6}$ and is briefly summarized as follows. If the mean flow through a duct with length $L$ is assumed to be uniform, and the sound field is confined to plane waves in the spanwise direction (between the opposing hard walls), the acoustic field propagating through the flow duct (assuming a time dependence of the form $\left.e^{i \omega t}\right)$ satisfies a convected Helmholtz equation on the acoustic pressure field

$$
\left(1-M^{2}\right) \frac{\partial^{2} p(x, y)}{\partial x^{2}}+\frac{\partial^{2} p(x, y)}{\partial y^{2}}-2 i k M \frac{\partial p(x, y)}{\partial x}+k^{2} p(x, y)=0
$$

where $p$ is the steady-state acoustic pressure field, $i=\sqrt{-1}$ is the unit imaginary number, $k=\omega / c$ is the freespace wavenumber, $\omega$ is the angular frequency, $c$ is the speed of sound in the duct, $M$ is the uniform flow Mach number, and $(x, y)$ are the coordinates in the axial and vertical directions, respectively. The normal component of the acoustic particle velocity vanishes at the rigid lower wall, which is given by

$$
\frac{\partial p(x, 0)}{\partial y}=0
$$

Source $(x=0)$ and exit $(x=L)$ plane boundary conditions are given by the measured acoustic pressures,

$$
p(0, y)=p(0,0) ; p(L, y)=p(L, 0)
$$

where $p(0,0)$ and $p(L, 0)$ are the measured acoustic pressures at the lower wall of the source and exit plane, respectively.

When written in terms of the acoustic pressure field, the locally-reacting wall boundary condition ${ }^{7}$ is given by

$$
-\frac{\partial p(x, H)}{\partial y}=i k\left(\frac{p(x, H)}{\zeta}\right)+2 M \frac{\partial}{\partial x}\left(\frac{p(x, H)}{\zeta}\right)+\frac{M^{2}}{i k} \frac{\partial^{2}}{\partial x^{2}}\left(\frac{p(x, H)}{\zeta}\right)
$$

where $H$ is the height of the duct and the normalized admittance, $1 / \zeta$, is taken as zero along the rigid wall portion of the upper wall. If the liner is uniform, this boundary condition is applied uniformly along the axial extent of the liner. For a two-segment liner, as is considered in this study, this boundary condition is 
separated into two components, where the first segment has an impedance of $\zeta_{1}$ and the second segment has an impedance of $\zeta_{2}$.

The CHE method of impedance eduction solves the boundary value problem defined in Eqs. (1)-(4) using a finite element method (FEM). The impedance boundary conditions are incorporated in the FEM analysis in weak form and cubic Hermite polynomials are used as the basis functions.

The unknown normalized impedances, $\zeta_{1}$ and $\zeta_{2}$, are educed by minimizing the objective function

$$
F\left(\zeta_{1}, \zeta_{2}\right)=\sum_{I=1}^{N}\left\|p\left(x_{I}, 0\right)_{\text {num }}-p\left(x_{I}, 0\right)_{\text {meas }}\right\|
$$

where $\|$ denotes the absolute value, $N$ is the number of microphones, $x_{I}$ is the location of the $I^{\text {th }}$ microphone, and the subscripts 'num' and 'meas' denote the numerically computed and measured pressure fields, respectively (note: $\zeta_{1}=\zeta_{2}$ for a uniform liner). Basically, this amounts to iteratively evaluating different values for the normalized impedances (all impedances in this paper are normalized by $\rho c$, where $\rho$ is the density of air in the duct) of each liner segment until this objective function is minimized.

A number of optimization algorithms have been considered for minimization of this objective function. For this study, Stewart's adaptation of the Davidon-Fletcher-Powell (SDFP) optimization algorithm ${ }^{8}$ is used with the uniform liners. This is the approach that has been used by NASA Langley for quite some time.

Three optimizers were considered for use with the two-segment liner, each implemented via Python. ${ }^{9}$ The first was a gradient-based optimizer that is very similar to the SDFP algorithm. When applied to the uniform liners, this optimizer provided very similar results to those achieved with the SDFP algorithm. However, due to the possibility of multiple local optima, especially as the number of axial segments was increased from one to two, two global optimizers (Basin-Hopping and Differential Evolution algorithms) were considered that are stochastic in nature and attempt to find the global minimum of a multivariate function. Both optimizers provided good results, but the Differential Evolution was more efficient (largely due to the particular settings used in the analysis). Thus, the results reported in this paper will be limited to those obtained with the Differential Evolution algorithm. This algorithm begins with a candidate population and with each iteration the algorithm mutates each candidate solution by mixing with other candidate solutions to create a trial candidate. The fitness of each trial candidate is assessed in relation to the objective function and the calculations continue until prescribed tolerances or maximum iterations are reached. This is the first time that the Differential Evolution algorithm has been implemented with the CHE indirect impedance eduction method.

\section{B. Kumaresan and Tufts (KT) Method}

The direct method of impedance eduction used in this study is based on the KT algorithm. Application of this method for liner impedance eduction has been described in an earlier paper. ${ }^{4}$ Therefore, only enough detail is presented here to motivate the methods and goals of the current investigation. To begin, the acoustic pressure field in the liner test section is expanded into a series of normal duct modes

$$
p(x, y)=\sum_{n=1}^{R} A_{n} P_{n}(y) e^{-i K_{n} x}, \quad P_{n}(y)=\cos \left(\lambda_{n} y\right), \quad \lambda_{n}^{2}=\left(k-K_{n} M\right)^{2}-K_{n}^{2}
$$

where $n$ is the mode order, $R$ is the total number of duct modes in the expansion, $A_{n}$ is the complex mode coefficient, $P_{n}(y)$ is the normal acoustic pressure mode, and $K_{n}$ is the axial propagation constant. Each term in the mode expansion [i.e., $A_{n} P_{n}(y) e^{-i K_{n} x}$ ] satisfies the convected Helmholtz equation [Eq. (1)] and the lower wall boundary condition [Eq. (2)]. Substituting the mode solution into the wall impedance boundary condition [Eq. (4)] gives an expression for the impedance

$$
\zeta=\frac{i k}{\lambda_{n}}\left(1-K_{n} M / k\right)^{2} \cot \left(\lambda_{n} H\right)
$$

In the direct method, $K_{n}$ is determined (via the KT algorithm) from the acoustic pressures measured with the microphones on the wall opposite the uniform-impedance liner (or liner segment). Equation (7) is then used to compute the unknown impedance, $\zeta$, of the test liner. 


\section{Experimental Method}

Three liner configurations are considered in this study. The first is a two-segment liner for which each segment consists of chambers with a unique depth. The other two have uniform depth for the entire liner. The depths of the two segments of the two-segment liner are chosen to match the depths of the two uniformdepth liners. Thus, the impedances educed for the two uniform-depth liners are expected to be very similar to those educed for each segement of the two-segment liner. This comparison is performed based on acoustic pressure data acquired with each liner mounted in the NASA LaRC grazing flow impedance tube (GFIT).

\section{A. Test Liners}

Three 2.0"-wide by 16.0"-long liner configurations, labeled as TL1, TL2, and TL3, are evaluated in this study. Based on CAD models of the liner channel geometry and depth distribution, each liner was built using a stereolithography (SLA) process whereby liquid resin is photo-polymerized using laser light to form the liner in 0.006 "-thick layers. The perforated facesheet was integrated with the core (i.e., it was included in the SLA fabrication process) while a solid backplate was used to seal the posterior of the liner. Finally, sanding was performed on the front and back surfaces to ensure flatness for the backplate and GFIT mounting shoulders. The as-built geometry was verified via measurement for later use in prediction models.

Figure 1 provides two top-view photographs of the TL3 liner. This liner consists of a $4 \times 30$ array of square $(0.4 " \times 0.4$ ") chambers separated by 0.135 "-wide axial partitions and 0.13 "-wide spanwise partitions. With the exception of chamber depth, all geometric parameters for the other two liners are identical to those for the TL3 liner. Each chamber consists of a 0.034"-thick perforated facesheet that contains 22 holes with a diameter of 0.041 ". This results in an overall porosity (including the effects of the partitions) of $11 \%$.

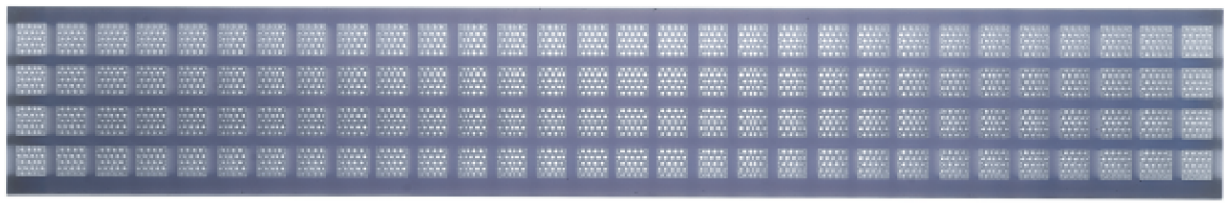

(a) Photo of liner.

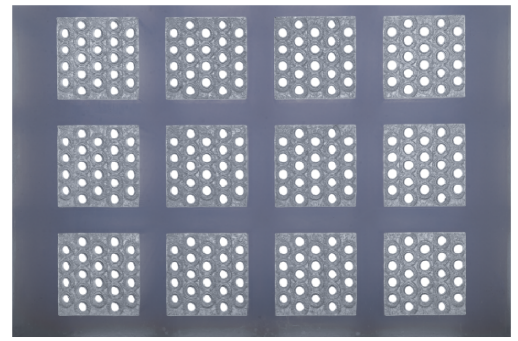

(b) Close-up photo of portion of liner.

Figure 1: Full length and close-up photos of test liner. Top view.

A side-view photograph of the TL1 and TL2 liners is provided in figure 2. The TL1 liner is divided into two axial segments. The first segment has an axial length of 10.7 " $(4 \times 20$ chambers $)$ and a uniform chamber depth of 2.0", while the second segment has a length of 5.3" (4×10 chambers) and a uniform chamber depth of 3.0". The TL2 and TL3 liners are uniform (all chambers are identical), with chamber depths of 2.0" and $3.0 "$ ", respectively.

\section{B. Grazing Flow Impedance Tube Measurements}

The NASA Langley grazing flow impedance tube (GFIT, see Fig. 3) has a cross-sectional geometry of 2.0" wide by 2.5 " high, and allows evaluation of acoustic liners with lengths from 2.0 to 24.0 " (16" length for 


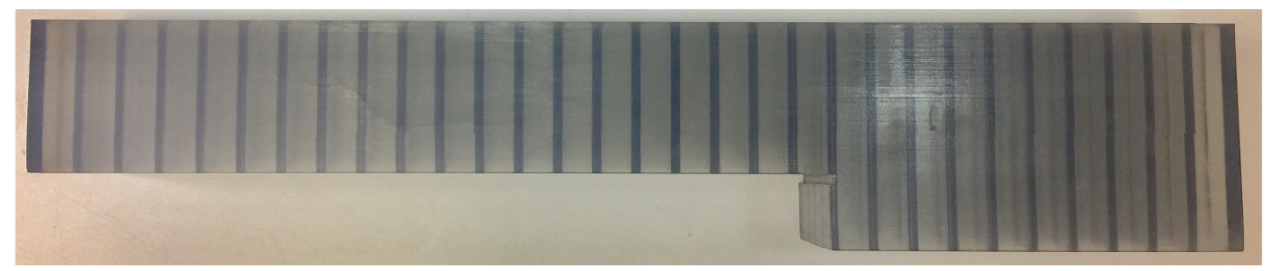

(a) 2-segment liner (TL1).

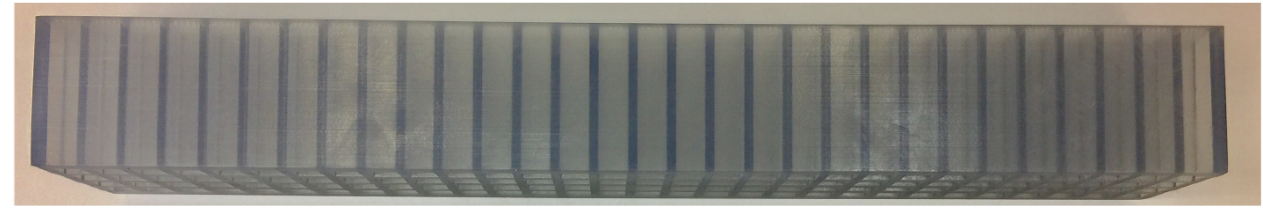

(b) Uniform liner with 2.0"-deep chambers (TL2).

Figure 2: Photographs of test liners. Side view.

the liners used in this study). The surface of the test liner forms a portion of the upper wall of the flow duct. Twelve acoustic drivers form an upstream (exhaust mode) source section. For this study, these drivers are used to generate tones (one frequency at a time) over a frequency range of 400 to $3000 \mathrm{~Hz}$ in $200 \mathrm{~Hz}$ increments, at source sound pressure levels (peak total SPL measured near the leading edge of the liner) of 120 and $140 \mathrm{~dB}$, and at centerline Mach numbers of 0.0 and 0.3. Fifty-three (53) microphones are flushmounted in the lower wall (opposite the liner) of the GFIT, and are used to measure the acoustic pressure field over the axial extent of 40 " (beginning 8" upstream of the liner leading edge). For the purposes of this study, the results are limited to those for which plane waves are cut on in the hardwall sections of the GFIT (i.e., up to $2400 \mathrm{~Hz}$ ).

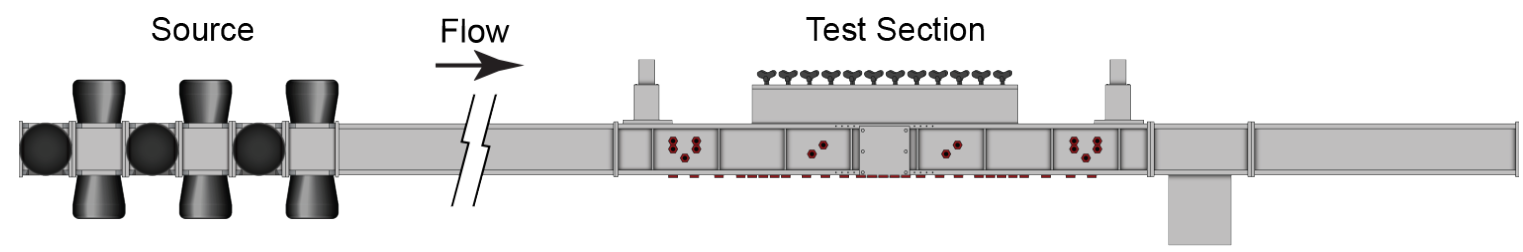

Figure 3: Sketch of NASA grazing flow impedance tube (GFIT).

\section{Results and Discussion}

Two independent methods are used to educe the impedance of the two-segment liner (TL1). The KT (direct) and CHE (indirect) methods are used to educe the impedance spectra for each of the two uniformdepth liners (TL2 and TL3), and the multisegment CHE method is used to educe the impedance spectra for each axial segment of the two-segment liner. Since the first segment of the TL1 liner has the same geometry as the TL2 uniform liner, it is expected that their respective impedance spectra should be identical. Similarly, it is expected that the second segment of the TL1 liner will have the same impedance as that provided by the uniform TL3 liner. Finally, the two-segment liner is also tested in the reverse orientation (labeled as TL1R), such that the 3"-deep chambers are at the leading edge.

Figure 4 compares four impedance spectra. The first two are impedances educed with the KT (red circles) and CHE (green squares) methods for the TL2 liner (uniform liner with 2" core depth). The third (blue diamonds) impedance is educed with the CHE method for the first segment (zone 1, 2" core depth) of the TL1 liner, and the last (dark red triangles) impedance is educed with the CHE method for the second segment (zone 2, 2" core depth) of the TL1 liner when placed in the reverse orientation (denoted as TL1R). 
There are a few minor outliers that are educed using the CHE method for the TL2 liner, but the comparison among the respective impedance spectra is quite favorable.

These results are attained with no flow and with a source level of $140 \mathrm{~dB}$. As noted earlier, tests were conducted at source levels of 120 and $140 \mathrm{~dB}$. However, since the results for each source SPL were very similar, the results presented in this report are limited to those acquired with a source SPL of $140 \mathrm{~dB}$. Also, it should be noted that the scales of the figures are chosen for uniformity throughout the ensuing data plots.

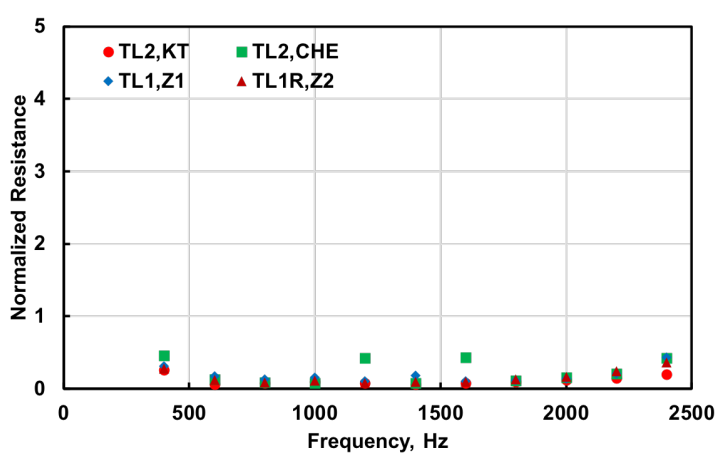

(a) Normalized resistance.

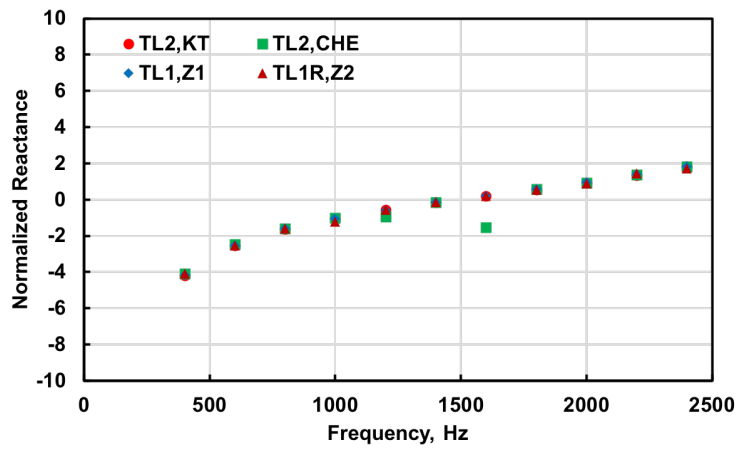

(b) Normalized reactance.

Figure 4: Comparison of impedance spectra educed for 2"-deep liner (TL2) or liner segment (zone 1 of TL1, zone 2 of TL1R). Mach 0.0, $140 \mathrm{~dB}$ source.

Figure 5 provides the corresponding results with the centerline Mach number set to 0.3. As expected, the normalized resistance increases by approximately 0.3 due to the presence of grazing flow. There is also a very slight flattening of the reactance spectrum (low frequency values increase and high frequency values decrease). There are a few more discrepancies between the respective results, but overall the comparison remains excellent.

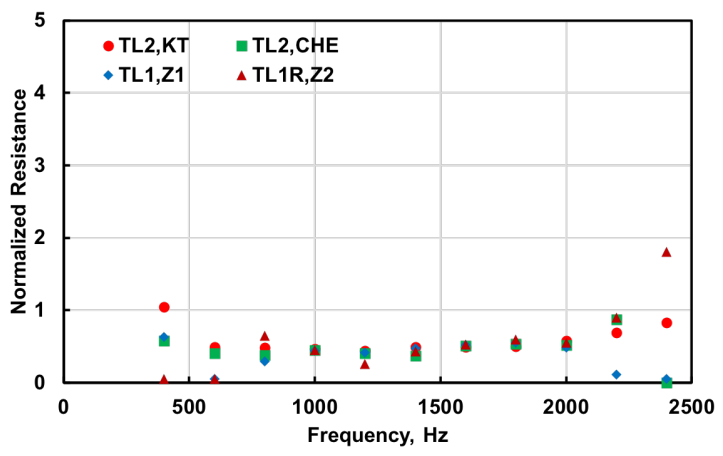

(a) Normalized resistance.

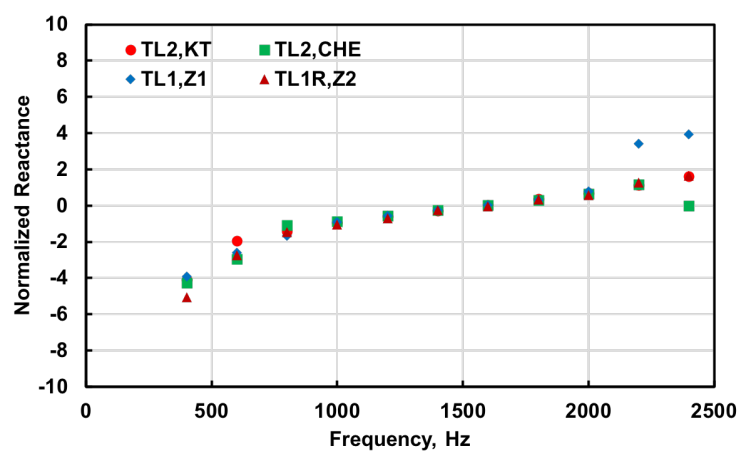

(b) Normalized reactance.

Figure 5: Comparison of impedance spectra educed for 2"-deep liner (TL2) or liner segment (TL1). Mach 0.3, $140 \mathrm{~dB}$ source.

Results for the 3"-deep liner (TL3) are compared with results for the 3"-deep segment of the TL1 liner (zone 2 for TL1, zone 1 for TL1R) in Figure 6, with no flow and a source SPL of $140 \mathrm{~dB}$. A few items are readily apparent in this figure. The resistance is generally quite similar to those for the 2"-deep liner. This is expected since the facesheet is identical for all liners used in this study. Also, the resistance increases at higher frequencies (off the scale for $2200 \mathrm{~Hz}$ ) due to the increase in magnitude of the reactance at these 
frequencies. This is caused by coupling between the resistance and reactance components. For this cavity depth, resonance occurs near $1100 \mathrm{~Hz}$, and antiresonance occurs near $2300 \mathrm{~Hz}$. Away from the antiresonance, the comparison remains favorable.

Taken together, the results presented in Figures 4, 5, and 6 indicate that the indirect impedance eduction method applied herein is appropriate for educing the impedance of multisegment liners. They also suggest that the effects of interactions between adjacent segments of the two-segment liner are minimal on the eduction process.

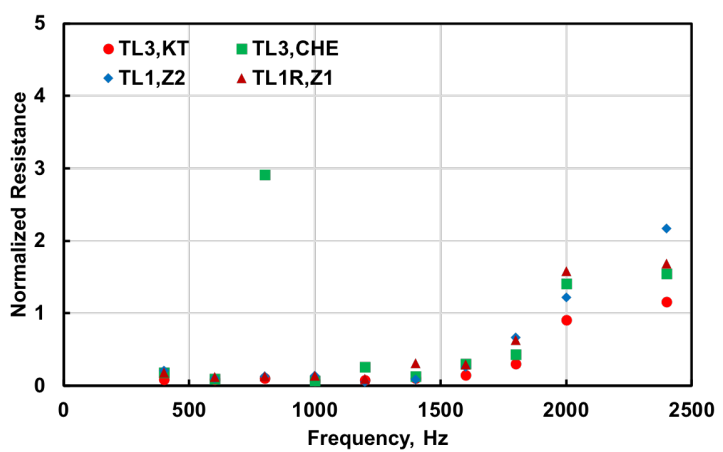

(a) Normalized resistance.

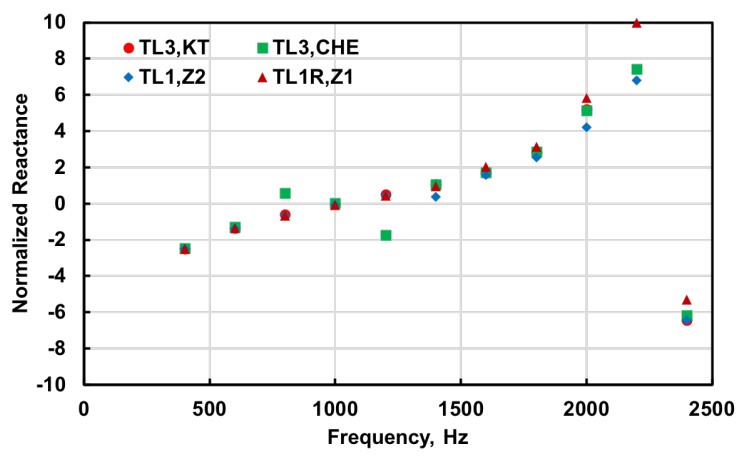

(b) Normalized reactance.

Figure 6: Comparison of impedance spectra educed for 3"-deep liner (TL3) or liner segment (TL1). Mach 0.0, $140 \mathrm{~dB}$ source.

Figure 7 presents the corresponding results at Mach 0.3. These results do not compare as favorably as those presented earlier. As noted in the results of Figure 5, the addition of grazing flow causes the resistance to increase and the reactance to flatten. At the lowest frequencies, the resistances educed for the second segment of the TL1 liner (TL1,Z2) are lower than those educed for the uniform 3"-deep liner (TL3) or for the first segment of the TL1 liner when it is tested in the reverse orientation (TL1R,Z1). At the higher frequencies, near antiresonance, it is the resistances educed for the latter case (TL1R,Z1) that deviate from the rest of the results. Overall, there is significantly more scatter in the results for frequencies above about $1500 \mathrm{~Hz}$.

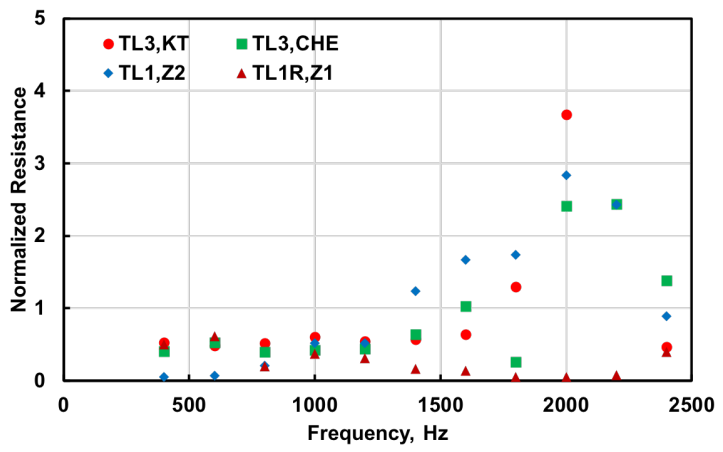

(a) Normalized resistance.

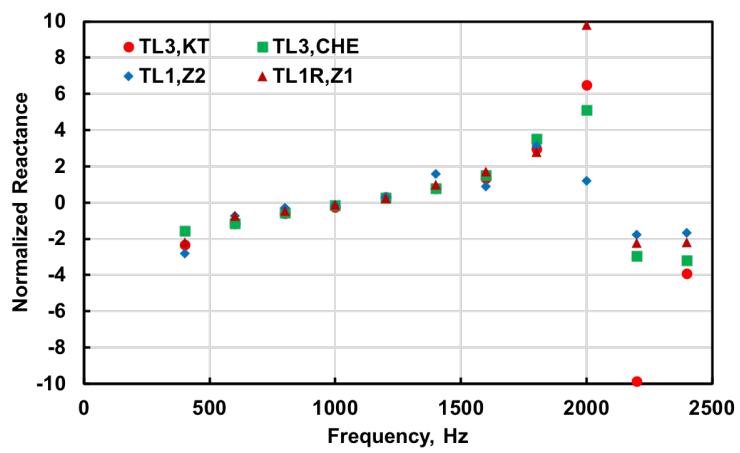

(b) Normalized reactance.

Figure 7: Comparison of impedance spectra educed for 3"-deep liner (TL3) or liner segment (TL1). Mach 0.3, $140 \mathrm{~dB}$ source.

Three reasons are offered to explain the divergence of results for the higher frequencies in this last case. First, it is likely that a further tightening of the tolerance for the optimizer would provide improved results. 
However, since the tolerance settings were not changed for each test in this study, and the results for the 2"-deep segment are excellent, other factors should be considered. Second, it is possible that there are interactive effects between the adjacent segments. This seems unlikely to be a dominant source of error since the results are much improved when the TL1 liner is tested in the original orientation (2"-deep chambers at the leading edge). The third reason, considered to be the leading candidate thus far, is the poor attenuation that is achieved for frequencies at or near antiresonance. This effect is clear in the axial acoustic pressure profiles presented in Figure 8 for the frequencies of 1600 to $2200 \mathrm{~Hz}$.

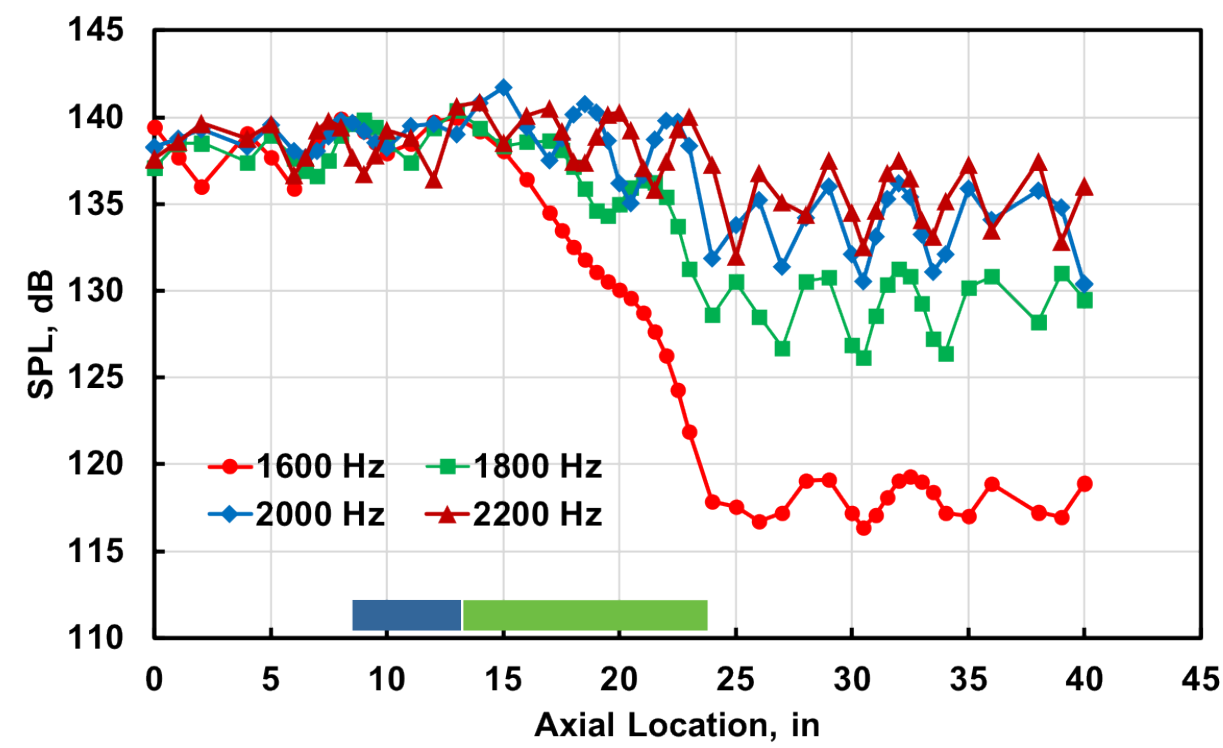

Figure 8: Axial acoustic pressure profiles for TL1 liner mounted in reverse orientation (TL1R). Mach 0.3, $140 \mathrm{~dB}$ source. Blue and green bars indicate the locations of the first (3" depth) and last (2" depth) segments of the liner when placed in this orientation.

Over the portion of the TL1 liner that is comprised of 3"-deep chambers (as indicated by the blue bar at the bottom of the plot), there is no attenuation. Instead, the acoustic pressure profiles are swamped by standing waves due to reflections from impedance discontinuities at the interface between segments, the end of the liner, or the termination of the GFIT. As the sound waves traverse into the second segment (2"-deep chambers for this configuration), the attenuation is increased. This explains why the results for the 2"-deep chambers presented in Figures 4 and 5 showed more favorable comparisons. Indeed, it is well known that impedance eduction methods are poorly suited for conditions where very limited attenuation is achieved. Fortunately, the results are of less interest in frequency regimes where the liner performs poorly.

\section{Concluding Remarks}

This paper explored the validity of an indirect method for impedance eduction of multisegmented liners. Two orientations of the two-segment liner were considered, one with 2"-deep chambers in the forward segment and 3"-deep chambers in the aft segment, and the other with this orientation reversed. Two uniform liners were also tested with identical geometrical parameters to those of the two distinct segments of the twosegment liner. The uniform liners were evaluated using direct (Kumaresan and Tufts) and indirect (Convected Helmholtz Equation finite element solver) methods to educe their respective acoustic impedance spectra. A two-segment indirect impedance eduction method (derivative of the indirect method used for uniform liners) was implemented to educe the impedances for each segment of the two-segment liner, and the results were compared with those educed for the uniform liners. While this is not a sufficiently thorough investigation to consider all possible issues with the proposed approach, the results are nonetheless quite encouraging. 
Some specific results are perhaps worthy of note. First, the results presented here suggest that interactive effects between adjacent segments of a multisegment liner are limited, at least in regard to the ability to educe the individual impedances for the respective segments. Second, the results presented here represent the first time that the Differential Evolution algorithm has been implemented via a Python-based optimizer for use with the CHE indirect impedance eduction method. This approach clearly warrants continued use. Finally, the multisegment impedance eduction approach implemented herein provides very good results, at least for the liners included in this study. As such, this tool offers the potential to study complicated liners in a more efficient manner, i.e., without the requirement to build and test separate liners to duplicate each unique segment of the multisegment liner. More detailed studies are planned to further validate this tool.

\section{Acknowledgements}

This work was funded by the Advanced Air Transport Technology Project of the NASA Advanced Air Vehicles Program. The support of Martha Brown and Alonso Reid for the acquisition of GFIT data is gratefully acknowledged.

\section{References}

\footnotetext{
${ }^{1}$ Jones, M. G., Watson, W. R., Nark, D. M., Schiller, N. H., and Born, J. C., "Optimization of Variable-Depth Liner Configurations for Increased Broadband Noise Reduction," AIAA Paper 2016-2783, May 2016.

${ }^{2}$ Jones, M. G., Nark, D. M., Watson, W. R., and Howerton, B. M., "Variable-Depth Liner Evaluation Using Two NASA Flow Ducts," AIAA Paper 2017-3022, June 2017.

${ }^{3}$ Dean, P. D., "An In Situ Method Of Wall Acoustic Impedance Measurement In Flow Ducts," Journal of Sound and Vibration, Vol. 34, No. 1, 1974, pp. 97-130.

${ }^{4}$ Watson, W. R., Carpenter, M. H., and Jones, M. G., "Performance of Kumaresan and Tufts Algorithm in Liner Impedance Eduction with Flow," AIAA Journal, Vol. 53, No. 4, April 2015, pp. 1091-1102.

${ }^{5}$ Watson, W. R. and Jones, M. G., "A Comparative Study of Four Impedance Eduction Methodologies Using Several Test Liners," AIAA Paper 2013-2274, May 2013.

${ }^{6}$ Watson, W. R., Jones, M. G., and Parrott, T. L., "Validation of an Impedance Eduction Method in Flow," AIAA Journal, Vol. 37, No. 7, July 1999, pp. 818-824.

${ }^{7}$ Myers, M. K., "On the Acoustic Boundary Condition in the Presence of Flow," Journal of Sound and Vibration, Vol. 71, No. 3, 1980, pp. 429-434.

${ }^{8}$ G. W. Stewart, I., "A Modification of Davidon's Minimization Method to Accept Difference Approximations of Derivatives," Journal of $A C M$, Vol. 14, No. 1, 1967, pp. 72-83.

${ }^{9}$ Jones, E., Oliphant, T., Peterson, P., et al., "SciPy: Open source scientific tools for Python," URL: http://www.scipy.org/, $2001-$.
} 